Published in final edited form as:

Acta Diabetol. 2018 September ; 55(9): 963-970. doi:10.1007/s00592-018-1181-4.

\title{
Relationship between sleep disturbance and self-care in adults with type 2 diabetes
}

\author{
Bingqian Zhu ${ }^{1,4}$, Laurie Quinn ${ }^{1}$, Mary C. Kapella ${ }^{1}$, Ulf G. Bronas ${ }^{1}$, Eileen G. Collins ${ }^{1}$, Laurie \\ Ruggiero $^{2,3}$, Chang G. Park ${ }^{1}$, Cynthia Fritschi ${ }^{1}$ \\ ${ }^{1}$ Department of Biobehavioral Health Science, College of Nursing, University of Illinois at \\ Chicago, 845 S. Damen Ave. (M/C 802), Chicago, IL 60612, USA \\ ${ }^{2}$ Institute for Health Research and Policy, School of Public Health, University of Illinois at Chicago, \\ Chicago, IL, USA \\ ${ }^{3}$ School of Nursing, University of Delaware, Newark, DE, USA \\ ${ }^{4}$ School of Nursing, Shanghai Jiaotong University, Shanghai, China
}

\begin{abstract}
Aims-Type 2 diabetes (T2D) is a metabolic disorder requiring intensive self-care to maintain optimal glycemic control. Sleep disturbance is common in T2D patients and likely impairs glycemic control. Our aim was to examine the relationship between sleep disturbance and self-care in adults with T2D.
\end{abstract}

Methods-This study used a correlational design. Sixty-four adults with T2D were recruited. Validated instruments were used to measure self-care, subjective sleep disturbance, and covariates (e.g., diabetes distress, self-efficacy, fatigue, and daytime sleepiness). Over an 8-day period, the ActiGraph-wGT3X was used to measure objective sleep outcomes (e.g., total sleep time and number of awakenings). Bivariate correlation and multiple linear regression analyses were conducted.

Results-The mean age of the participants was 60.6 (SD 6.8) years (range 50-78), and 51.6\% were women. Controlling for covariates, subjective sleep disturbance $(\beta=-0.26)$, diabetes distress $(\beta=-0.39)$, and daytime sleepiness $(\beta=-0.21)$ were strong predictors of diabetes selfcare $\left(R^{2}=0.51, p<0.001\right)$. When objective sleep parameters were used, the number of awakenings $(\beta=-0.23)$ also predicted self-care along with diabetes distress, fatigue, and daytime sleepiness $\left(R^{2}=0.57, p<0.001\right)$.

Conclusions-Subjective sleep disturbance and frequent nocturnal awakenings are associated with worse diabetes self-care in adults with T2D. Healthcare providers are recommended to include comprehensive sleep assessment at every clinical visit. Diabetes educators may consider

Cynthia Fritschi, fritschi@uic.edu.

Conflict of interest The authors declare that they have no conflict of interest.

Ethical approval All procedures performed in this study were in accordance with the ethical standards of the institutional and/or national research committee and with the 1964 Helsinki Declaration and its later amendments or comparable ethical standards.

Informed consent Informed consent was obtained from all individual participants included in the study. 
including sleep-related education in their diabetes self-management classes and/or counseling sessions.

\section{Keywords}

Actigraphy; Nocturnal awakening; Self-care; Sleep; Symptom; Type 2 diabetes

\section{Introduction}

Diabetes self-care behaviors are daily, lifelong, complex regimen tasks that an individual performs to manage diabetes [1]. Self-care is the foundation for optimal glycemic control, which is crucial for preventing or delaying chronic diabetic complications [2], such as early disability, amputation, and kidney failure. Chronic complications have been estimated to account for $53 \%$ of the total amount of diabetes-related expenditure [3]. Diabetes self-care is often challenging for aging adults and is further complicated by competing psychosocial and physiological burdens [4], among which sleep disturbance might play a role.

Sleep disturbance is a frequent complaint in people with type 2 diabetes (T2D), with the prevalence ranging from 42 to $76.8 \%$ [5, 6]. Aging adults with T2D are more vulnerable to sleep disturbance due to age-related physiological changes and comorbidities [7]. Sleep disturbance has been related to impaired glycemic control [8], yet the relationship between sleep disturbance and self-care has been under-examined. Findings from earlier crosssectional studies of sleep disturbance and self-care have been inconclusive. Chasens and colleagues reported that self-reported sleep disturbance was associated with control problems, attitude, and self-care adherence [9]. In contrast, Nefs and colleagues [6] found no significant relationship between self-reported sleep disturbance and self-care in a crosssectional study. Prior studies may have been limited with the use of static, cross-sectional measures of sleep and bivariate analysis. There is a need to examine whether sleep disturbance is related to daily self-care behaviors in the context of aging adults with T2D while addressing those methodological limitations.

Several factors may need to be controlled while examining the relationship between sleep disturbance and self-care. These factors include self-efficacy, diabetes distress, fatigue, and daytime sleepiness. Evidence has shown that self-efficacy is a significant and unique predictor of self-care behaviors in people with T2D [10]. Similarly, diabetes distress has been a strong predictor of self-care [11]. As a common and debilitating symptom, fatigue is associated with self-care [12]. Daytime sleepiness, one's tendency to fall asleep under different situations [13], has also been related to self-care [9].

Building upon previous studies, we aimed to examine the relationship between sleep disturbance and self-care. We included both subjective and objective sleep assessment and controlled for potential covariates. Understanding the relationship between sleep disturbance and diabetes self-care may contribute to our knowledge in the complex relationship between sleep and diabetes. Findings from this study may help to improve current diabetes care guidelines by further encouraging sleep-related assessment and intervention. 


\section{Participants and methods \\ Design}

We used a correlational design to study the relationships between sleep and self-care behaviors at baseline and over an 8-day period under free-living conditions.

\section{Participants}

A convenience sample of adults (aged 50 years or over) with T2D for over a year was recruited. Participants with the following self-reported conditions were excluded: (1) gestational diabetes; (2) on anti-depression therapy, anxiolytic agents, and antipsychotics, or with other physical disabilities (e.g., amputation and paralysis); (3) acute diabetic complications or other comorbidities including chronic arthritis with uncontrolled pain, cancer within the past year, heart failure, liver disease, chronic obstructive pulmonary disease, and kidney failure; (4) using prescription sleep medications for insomnia or diagnosed with restless leg syndrome; (5) shift-workers; and (6) non-English speakers.

Baseline data from the first 50 participants were used to run a simulation-based power analysis [14]. The simulation results suggested that with a sample size of 50, the power of detecting a significant relationship between subjective sleep disturbance and self-care was 0.78 . When the sample size is increased to 60 , the anticipated power is 0.88 , suggesting that a sample size of 60 should be large enough to detect the significance. A total of 64 participants were recruited in this study, and 59 completed that 8-day data collection. The recruitment process is shown in Fig. 1.

\section{Measurements}

Demographic and health-related characteristics-Participant demographic and health-related characteristics were measured using a baseline questionnaire designed by the researchers. The questionnaire included self-reported: age, gender, race/ethnicity, education years, diabetes duration, treatment regimen, hypertension, and diagnosis of sleep apnea. Fingerstick capillary-glycated hemoglobin A1c (HbA1c) was collected using Bayer A1CNow ${ }^{\mathrm{TM}}[15]$. Height and weight were measured using a wall-mounted stadiometer and upright, balanced scale, respectively.

Self-care-The Diabetes Self-Management Questionnaire-Revised (DSMQ-R) [16] was used to assess diabetes self-care over the prior 2 months. The DSMQ-R consists of four subscales: glucose management, dietary control, physical activity, and physician contact. Each item evaluates an individual's perception of his/her self-care behaviors using a 4-point Likert scale. The sum of all items yields a global score, which is transformed to a scale ranging from 0 to 10. A higher score indicates better self-care. The DSMQ has acceptable internal consistency reliability (Cronbach's $a=0.82$ ) in adults with T2D [16]. In this sample, the Cronbach's $a$ of the DSMQ-R was 0.79 .

Subjective sleep disturbance-The Pittsburgh Sleep Quality Index (PSQI) [17] was used to measure subjective sleep quality and sleep disturbance over the past month. The PSQI consists of 19 items, forming seven factors: subjective sleep quality, sleep duration, 
sleep latency, sleep efficiency, sleep disturbance, use of sleep medication, and daytime dysfunction. The PSQI global score ranges from 0 to 21. A higher score means poorer sleep quality and a global score of over 5 indicates sleep disturbance. The PSQI has acceptable internal consistency (Cronbach's $a=0.83$ ) and test-retest reliability. It also showed high sensitivity (89.6\%), specificity (86.5\%), and accuracy (88.5\%) when a cut-off point was set at 5 , supporting its ability to discriminate those with and without sleep disturbance [17]. The Cronbach's $a$ was 0.71 in this sample.

Objective sleep-ActiGraph wGT3X was used to measure objective sleep. The ActiGraph wGT3X is a wrist watch-like device that records activity information using a triaxial accelerometer. Data were collected in the $30 \mathrm{~s}$ epoch, and each epoch of data was evaluated as sleep or wake, based on the specific algorithm [18]. When compared to eight other wearable devices, the ActiGraph GT3X had the closest measure of sleep [19]. ActiGraph GT3X also showed good agreement with the actiwatch for assessing sleep [20]. When tested against PSG in healthy adults, the wrist-worn ActiGraph wGT3X has a high sensitivity (90\%) and accuracy (84\%), whereas low specificity (46\%) [21]. The Cole-Kripke algorithm [22] embedded in Actilife 6 (ActiGraph, Pensacola, FL) was used to score the actigraphy following standard procedures [23]. In this study, the sleep parameters included total sleep time (TST), sleep efficiency (SE), sleep onset latency (SOL), wake after sleep onset (WASO), and the number of awakenings.

Self-efficacy-The Diabetes Empowerment Scale-Short Form (DES-SF) [24] was used to assess self-efficacy. The DES-SF is an 8-item scale assessing the overall diabetes-related psychosocial self-efficacy. Each item is rated on a 5-point Likert scale. The average of the eight items results in the total score. A higher score indicates a higher level of self-efficacy. The DES-SF has good internal consistency reliability (Cronbach's $a>0.84$ ) [24]. In this sample, the Cronbach's $a$ was 0.83 .

Diabetes distress-The Diabetes Distress Scale (DDS) [25] was used to measure diabetes distress. The DDS consists of 17 items that measure four domains of diabetesrelated emotional distress over the past month: Emotional Burden, physician-related distress, regimen-related distress, and diabetes-related intrapersonal distress. Each item is scored on a 6-point Likert scale. A higher score indicates a greater level of distress. The DDS has high internal consistency reliability (Cronbach's $a>0.87$ ) [25]. In this sample, the Cronbach's $a$ of the DDS was 0.94 .

Fatigue-The fatigue subscale of the Diabetes Symptom Checklist-Revised (DSC-R) [26] was used to measure fatigue. The DSC-R measures perceived occurrence and burden of T2D-related physical and psychological symptoms during the past month. It consists of 34 items that measure eight domains including fatigue. Each item is rated on a 5-point Likert scale (1-not at all, 5-extremely). The domain score is the sum of domain items, and a higher score indicates greater symptom burden. The Cronbach's $a$ for the fatigue subscale was 0.87 [26], supporting its reliability. In this sample, the Cronbach's a for the fatigue subscale was 0.83 . 
Daytime sleepiness-The Epworth Sleepiness Scale (ESS) [27] was used to measure sleepiness. The ESS contains eight items that assess one's tendency to fall asleep in eight situations. Each item is rated on a 4-point Likert scale. The sum of the eight items results in a total score, ranging from 0 to 24 . A higher score indicates a higher level of sleepiness. Reliability of the ESS was supported by its good internal consistency (Cronbach's $a=0.88$ ) and high test-retest correlation ( $r=0.82)$ [27]. In this sample, the Cronbach's $a$ was 0.73 .

\section{Procedures}

The study was approved by the Institutional Review Board of the University of Illinois at Chicago. Participants were recruited through electronic flyers posted on campus mailing list and internet. Previous participants with T2D who have given permission to be contacted for future research were also contacted.

Prior to data collection, written informed consent was obtained. Baseline data were collected at a large urban university in the Midwestern of the United States. Validated questionnaires were administered during the baseline visit. During the following 8-day period, participants were instructed to wear the ActiGraph wGT3X on the non-dominant wrist and fill out the daily sleep diary within 1 hour upon awakening. Each diary was sent via REDCap electronic data capture tools hosted at the University of Illinois at Chicago [28].

\section{Statistical analysis}

Stata 13.0 (StataCorp LP, College Station, Texas) was used for statistical analyses. Prior to data analysis, missing data, normal distribution, and outliers were checked. Descriptive statistics $(\bar{x} \pm$ SD or percentage) were examined. Pearson's correlation analyses were used to examine the correlations between continuous variables. An extended actigraphy sleep assessment (5 days or longer) can reduce inherent measurement errors and increases reliability [18]. Therefore, only participants who wore the ActiGraph for 5 days or over (including at least 1 day of the weekend) were included in the analysis. Averages of each objective sleep variable were used in the analyses. Multiple linear regression analysis was run to examine the relationship between sleep and self-care. Covariates entered into the regression models were chosen based on the current literature and bivariate results. Variables that were significantly related to self-care at $p<0.2$ were included in the model. The following four assumptions were examined: model specification, homoscedasticity, multicollinearity, and normal distribution of residuals. Statistical significance was set at $p<$ 0.05 .

\section{Results}

\section{Sample characteristics}

Demographic and health-related characteristics of the participants are listed in Table 1. The sample included 64 participants (age $60.6 \pm 6.8$ years, 51\% female, 53\% African American). The mean duration of diabetes was $11.2 \pm 8.8$ years and mean $\mathrm{HbA1c}$ level was $7.9 \pm 2.0 \%$ $(63 \pm 22 \mathrm{mmol} / \mathrm{mol})$. Over half of the participants $(61.9 \%)$ did not achieve the goal for optimal glycemic control defined by the American Diabetes Association [HbA1c $<7.0 \%$ (53 $\mathrm{mmol} / \mathrm{mol})][29]$. 


\section{Description of main study variables}

The description of main study variables is presented in Table 2. Overall, participants mean DSMQ-R score was 6.9 (SD 1.5), ranging from 2.5 to 10. Participants mean PSQI global score was 7.0 (SD 3.7), and 54.7\% had subjective sleep disturbance (PSQI > 5).

\section{Bivariate correlations between self-care and other variables}

Table 3 shows the bivariate correlations between self-care and other variables of interest. Self-care was negatively related to subjective sleep disturbance $(r=-0.36, p<0.01)$. Among objective sleep measures, the number of awakenings was negatively related to selfcare $(r=-0.36, p<0.01)$.

\section{Linear regression model predicting self-care from subjective sleep disturbance}

Collectively, age, diabetes duration, sleep disturbance, self-efficacy, diabetes distress, fatigue, and daytime sleepiness explained $51 \%$ of the variations in self-care. Sleep disturbance, diabetes distress, and daytime sleepiness were significant predictors. A one-unit increase in the PSQI global score was related to 0.10 unit decrease in DSMQ-R (Table 4 model 1).

\section{Linear regression model predicting self-care from objective sleep}

Collectively, age, diabetes duration, number of awakenings, self-efficacy, diabetes distress, fatigue, and daytime sleepiness explained $57 \%$ of the variations in self-care. Number of awakenings, diabetes distress, fatigue, and daytime sleepiness were significant predictors. A one-unit increase in the number of awakening was associated with 0.05 -unit decrease in DSMQ-R (Table 4, model 2).

\section{Discussion}

The aim of this study was to examine the relationship between sleep disturbance and diabetes self-care. In this study, we systematically investigated this relationship among adults with T2D. We included both subjective and objective assessment of sleep and controlled for potential covariates. We found that subjective sleep disturbance and objective number of awakenings were associated with diabetes self-care. Findings from this study contributed to our knowledge in the complex relationship between sleep and diabetes.

In this study, subjective sleep disturbance was related to diabetes self-care. The bivariate correlation analysis suggested that sleep disturbance was significantly related to diabetes overall self-care $(r=-0.36)$. This finding is consistent with the previous results. In community-dwelling T2D adults with excessive daytime sleepiness (ESS > 10), sleep disturbance was associated with self-care adherence as measured by the Diabetes Care Profile ( $r=-0.214)$ [9]. In another study [6] consisting of 361 T2D adults, Nefs and colleagues reported that, compared to those without sleep disturbance, those with sleep disturbance (PSQI > 5) had suboptimal self-care. However, potential covariates were not controlled in prior studies, which may have resulted in biased results. In contrast, we obtained a more robust estimation by controlling for potential covariates, such as diabetes distress and daytime sleepiness. Diabetes distress has been widely recognized as a strong 
predictor of self-care [11,30], and our regression analysis revealed similar findings. Additionally, we found that sleep disturbance $(\beta=-0.26)$ was also a strong predictor of diabetes self-care. Accumulating evidence indicates that daytime sleepiness is related to diabetes self-care [9, 31]. Previous studies [32, 33] suggest that sleep disturbance may impair self-care through its effect on daytime sleepiness. However, in the present study, we demonstrated the independent effect of sleep disturbance and daytime sleepiness on selfcare. The relationship between sleep disturbance and diabetes self-care was even stronger than the relationship between the commonly reported daytime sleepiness and self-care.

Sleep continuity (infrequent nocturnal awakenings) is a hallmark of good sleep [34]. Aging adults [35, 36] and diabetes patients [35] are at particular risk for frequent nocturnal awakenings. In this study, the average number of nightly awakening derived from actigraphy was 18.7 (SD 7.2). Importantly, the number of awakenings was significantly related to selfcare. Limited studies have examined the relationship between nocturnal awakenings and self-care. Self-reported number of awakenings has been related to daytime functioning, such as difficulty concentrating, memory problems, and mood [35]. Frequent nocturnal awakenings result in fragmented sleep, which could impair cognitive functions through changes in sleep dynamics and constant disruptions of the normal sleep process [37]. In a randomized, cross-over study [38], 15 healthy males underwent artificially fragmented sleep. Fragmented sleep resulted in a higher level of daytime sleepiness, compared with the nonfragmented sleep. This finding suggests that fragmented sleep may cause sleepiness, and thereby influence daytime self-care behaviors. Another review [39] further supports that fragmented sleep may lead to sleepiness-related daytime impairment. However, we observed an independent effect of nocturnal awakenings on the self-care in this population.

Multiple physiological pathways have been proposed to explain the relationship between sleep disturbance and diabetes. Such pathways include decreased brain glucose utilization, increased sympathetic system activity, increased inflammation, abnormal adipocyte function, and alteration in appetite-regulating hormones caused by sleep disturbance [40, 41]. In addition to these physiological pathways, behavioral mechanisms linking sleep disturbance and diabetes have been suggested $[42,43]$. Previous studies conducted in patients with heart failure also indicated that sleep disturbance might affect self-care through its effect on mood, cognitive function, and functional performance $[32,33]$. While we did not measure those variables, we did include a measure of diabetes distress. Our findings provided supportive evidence for the complex relationship between sleep and self-care.

Subjective and objective measures of sleep provided different perspectives on an individual's sleep. One strength of our study is the inclusion of both methods, which enabled us to examine participants' sleep more thoroughly than the use of a single measure. Additionally, objective sleep was measured in the natural setting over multiple days, which yielded a more valid and reliable assessment. We also controlled for several potential confounding variables, which made our findings more robust. However, study findings need to be interpreted in light of the limitations. Causality between sleep and self-care can- not be inferred due to the correlational design. Generalizability of the study is limited to midlife and aging adults with T2D. Additionally, we used the Actigraph manufacturer's default scoring algorithm to produce objective sleep variables. Although the algorithm was developed and validated in 
adults, a different algorithm might be needed specifically for older adults with T2D. Future studies with a more representative sample are warranted to confirm the findings of the present study.

In conclusion, we demonstrated that sleep disturbance and more nocturnal awakenings are associated with lower levels of diabetes self-care in this group of adults with T2D. These findings have important implications for both research and clinical practice. Experimental studies are warranted to examine the impact of sleep interventions on diabetes self-care. Ideally, these interventions should target improving overall sleep quality and reducing nocturnal awakenings. Diabetes self-care consists of multiple and distinct behaviors, such as physical activity and eating behaviors. More studies are needed to investigate how sleep disturbance might affect each of these different behaviors.

Study findings can be easily translated into practice. Sleep evaluation has been recommended by the American Diabetes Association guideline [44]. Our findings add to evidence of including sleep-related assessment and intervention in the guideline, particularly for older adults. That would increase diabetes educators' and clinicians' awareness of the importance of sleep in self-care behavior. In clinical practice, healthcare providers are recommended to include comprehensive sleep assessment at every clinical visit. Diabetes educators may consider including sleep-related education in their diabetes self-management classes and/or counseling sessions.

\section{Acknowledgements}

This research was supported by the Midwest Roybal Center for Health Promotion and Translation from the National Institute on Aging (Grant\# P30AG022849). This research was also supported by the Institute for Health Research and Policy Grant support [Center for Clinical and Translational Science (CCTS) UL1RR029879], the Chancellor's Graduate Research Award, and the Provost Award for Graduate Research of the University of Illinois at Chicago. This research was also supported by the National Institutes of Health/National Institute for Nursing Research and Department of Veterans Affairs, Research Career Scientist Award (EC) [K99 R00 NR012219 (CF)].

Funding The funding source had no role in the design, data collection, analysis or interpretation of the study or in the decision to submit the manuscript for publication. We thank all participants for participating in this research.

\section{References}

1. Weinger K, Butler HA, Welch GW, La Greca AM (2005) Measuring diabetes self-care. Diabetes Care 28(6):1346-1352 [PubMed: 15920050]

2. American Diabetes Association (2015) Standards of medical care in diabetes-2015 abridged for primary care providers. Clin Diabetes 33 (2):97 [PubMed: 25897193]

3. Zhuo X, Zhang P, Hoerger TJ (2013) Lifetime direct medical costs of treating type 2 diabetes and diabetic complications. Am J Prev Med 45(3):253-261 [PubMed: 23953350]

4. Beverly EA, Ritholz MD, Shepherd C, Weinger K (2016) The psychosocial challenges and care of older adults with diabetes:"can't do what I used to do; can't be who I once was”. Curr Diab Rep 16(6):48 [PubMed: 27085863]

5. Gupta S, Wang Z (2016) Predictors of sleep disorders among patients with type 2 diabetes mellitus. Clin Res Rev 10(4):213-220

6. Nefs G, Donga E, van Someren E et al. (2015) Subjective sleep impairment in adults with type 1 or type 2 diabetes: results from diabetes MILES—The Netherlands. Diabetes Res Clin Pract 109(3): 466-475 [PubMed: 26264411]

7. Ancoli-Israel S (2009) Sleep and its disorders in aging populations. Sleep Med 10:S7-S11 [PubMed: 19647483] 
8. Lee SWH, Ng KY, Chin WK (2017) The impact of sleep amount and sleep quality on glycemic control in type 2 diabetes: a systematic review and meta-analysis. Sleep Med Rev 31:91-101 [PubMed: 26944909]

9. Chasens ER, Korytkowski M, Sereika SM, Burke LE (2013) Effect of poor sleep quality and excessive daytime sleepiness on factors associated with diabetes self-management. Diabetes Educ 39(1):74-82 [PubMed: 23192600]

10. Lee Y, Shin S, Wang R, Lin K, Lee Y, Wang Y (2016) Pathways of empowerment perceptions, health literacy, self-efficacy, and self-care behaviors to glycemic control in patients with type 2 diabetes mellitus. Patient Educ Couns 99(2):287-294 [PubMed: 26341940]

11. Pintaudi B, Lucisano G, Gentile S, Bulotta A, Skovlund SE, Vespasiani G, Rossi MC, Nicolucci A, BENCH-D Study Group (2015) Correlates of diabetes-related distress in type 2 diabetes: findings from the benchmarking network for clinical and humanistic outcomes in diabetes (BENCH-D) study. J Psychosom Res 79(5):348-354 [PubMed: 26526307]

12. Kirk JK, Arcury TA, Ip E, Bell RA, Saldana S, Nguyen HT, Quandt SA (2015) Diabetes symptoms and self-management behaviors in rural older adults. Diabetes Res Clin Pract 107(1):54-60 [PubMed: 25467626]

13. Shen J, Barbera J, Shapiro CM (2006) Distinguishing sleepiness and fatigue: focus on definition and measurement. Sleep Med Rev 10(1):63-76 [PubMed: 16376590]

14. Kontopantelis E, Springate D, Parisi R, Reeves D (2016) Simulation-based power calculations for mixed effects modelling: Ipdpower in Stata. J Stat Softw 74(12):1-25

15. Bode BW, Irvin BR, Pierce JA, Allen M, Clark AL (2007) Advances in hemoglobin A1c point of care technology. J Diabetes Sci Technol 1(3):405-411 [PubMed: 19885097]

16. Schmitt A, Gahr A, Hermanns N, Kulzer B, Huber J, Haak T (2013) The Diabetes SelfManagement Questionnaire (DSMQ): development and evaluation of an instrument to assess diabetes self-care activities associated with glycaemic control. Health Qual Life Outcomes 11(1): 138-151 [PubMed: 23937988]

17. Buysse DJ, Reynolds CF III, Monk TH, Hoch CC, Yeager AL, Kupfer DJ (1991) Quantification of subjective sleep quality in healthy elderly men and women using the Pittsburgh Sleep Quality Index (PSQI). Sleep 14(4):331-338 [PubMed: 1947597]

18. Sadeh A (2011) The role and validity of actigraphy in sleep medicine: an update. Sleep Med Rev 15(4):259-267 [PubMed: 21237680]

19. Rosenberger ME, Buman MP, Haskell WL, McConnell MV, Carstensen LL (2016) Twenty-four hours of sleep, sedentary behavior, and physical activity with nine wearable devices. Med Sci Sports Exerc 48(3):457-465 [PubMed: 26484953]

20. Cellini N, McDevitt EA, Mednick SC, Buman MP (2016) Free-living cross-comparison of two wearable monitors for sleep and physical activity in healthy young adults. Physiol Behav 157:7986 [PubMed: 26821185]

21. Slater JA, Botsis T, Walsh J, King S, Straker LM, Eastwood PR (2015) Assessing sleep using hip and wrist actigraphy. Sleep Biol Rhythms 13(2):172-180

22. Cole RJ, Kripke DF, Gruen W, Mullaney DJ, Gillin JC (1992) Automatic sleep/wake identification from wrist activity. Sleep 15(5):461-469 [PubMed: 1455130]

23. Ancoli-Israel S, Martin JL, Blackwell T, Buenaver LF, Liu L, Meltzer LJ, Sadeh A, Spira AP, Taylor DJ (2015) The SBSM guide to actigraphy monitoring: clinical and research applications. Behav Sleep Med 13(sup1):S4-S38 [PubMed: 26273913]

24. Anderson RM, Fitzgerald JT, Gruppen LD, Funnell MM, Oh MS (2003) The diabetes empowerment scale-short form (DES-SF). Diabetes Care 26(5):1641-1642

25. Polonsky WH, Fisher L, Earles J, Dudl RJ, Lees J, Mullan J, Jackson RA (2005) Assessing psychosocial distress in diabetes development of the diabetes distress scale. Diabetes Care 28(3): 626-631 [PubMed: 15735199]

26. Arbuckle RA, Humphrey L, Vardeva K, Arondekar B, Danten-Viala MSc M, Scott JA, Snoek FJ (2009) Psychometric evaluation of the diabetes symptom checklist-revised (DSC-R)—A measure of symptom distress. Value Health 12(8):1168-1175 [PubMed: 19558371]

27. Johns MW (1991) A new method for measuring daytime sleepiness: the Epworth Sleepiness Scale. Sleep 14(6):540-545 [PubMed: 1798888] 
28. Harris PA, Taylor R, Thielke R, Payne J, Gonzalez N, Conde JG (2009) Research electronic data capture (REDCap) — a metadata-driven methodology and workflow process for providing translational research informatics support. J Biomed Inform 42(2):377-381 [PubMed: 18929686]

29. American Diabetes Association (2017) Standards of medical care in diabetes: glycemic targets. Diabetes Care 40 (Suppl. 1):S48-S56 [PubMed: 27979893]

30. Pandit AU, Bailey SC, Curtis LM, Seligman HK, Davis TC, Parker RM, Schillinger D, DeWalt D, Fleming D, Mohr DC (2014) Disease-related distress, self-care and clinical outcomes among lowincome patients with diabetes. J Epidemiol Community Health 68(6):557-564 [PubMed: 24489044]

31. Chasens ER, Olshansky E (2008) Daytime sleepiness, diabetes, and psychological well-being. Issues Ment Health Nurs 29(10):1134-1150 [PubMed: 18853351]

32. Riegel B, Weaver TE (2009) Poor sleep and impaired self-care: towards a comprehensive model linking sleep, cognition, and heart failure outcomes. Eur J Cardiovasc Nurs 8(5):337-344 [PubMed: 19679510]

33. Redeker NS (2008) Sleep disturbance in people with heart failure: implications for self-care. J Cardiovasc Nurs 23(3):231-238 [PubMed: 18437065]

34. Libman E, Fichten C, Creti L, Conrod K, Tran D, Grad R, Jorgensen M, Amsel R, Rizzo D, Baltzan M (2016) Refreshing sleep and sleep continuity determine perceived sleep quality. Sleep Disord 2016:1-10

35. Ohayon MM (2010) Nocturnal awakenings and difficulty resuming sleep: their burden in the European general population. J Psychosom Res 69(6):565-571 [PubMed: 21109044]

36. Crowley K (2011) Sleep and sleep disorders in older adults. Neuropsychol Rev 21(1):41-53 [PubMed: 21225347]

37. Short MA, Banks S (2014) The functional impact of sleep deprivation, sleep restriction, and sleep fragmentation In: Bianchi MT (ed) Sleep deprivation and disease: effects on the body, brain and behavior. Springer, New York, pp 13-26

38. Hursel R, Rutters F, Gonnissen HKJ, Martens EAP, Westerterp-Plantenga MS (2011) Effects of sleep fragmentation in healthy men on energy expenditure, substrate oxidation, physical activity, and exhaustion measured over $48 \mathrm{~h}$ in a respiratory chamber. Am J Clin Nutr 94(3):804-808 [PubMed: 21795436]

39. Stepanski EJ (2002) The effect of sleep fragmentation on daytime function. Sleep 25(3):268-276 [PubMed: 12003157]

40. Reutrakul S, Van Cauter E (2014) Interactions between sleep, circadian function, and glucose metabolism: implications for risk and severity of diabetes. Ann N Y Acad Sci 1311(1):151-173 [PubMed: 24628249]

41. Martins RC, Andersen ML, Tufik S (2008) The reciprocal interaction between sleep and type 2 diabetes mellitus: facts and perspectives. Braz J Med Biol Res 41(3):180-187 [PubMed: 18060321]

42. Larcher S, Benhamou P-Y, Pépin J-L, Borel A-L (2015) Sleep habits and diabetes. Diabetes Metab 41(4):263-271 [PubMed: 25623152]

43. Grandner MA, Seixas A, Shetty S, Shenoy S (2016) Sleep duration and diabetes risk: Population trends and potential mechanisms. Curr Diab Rep 16(11):106 [PubMed: 27664039]

44. American Diabetes Association (2018) Standards of medical care in diabetes: comprehensive medical evaluation and assessment of comorbidities. Diabetes Care 41 (Suppl. 1):S28-S37 [PubMed: 29222374] 


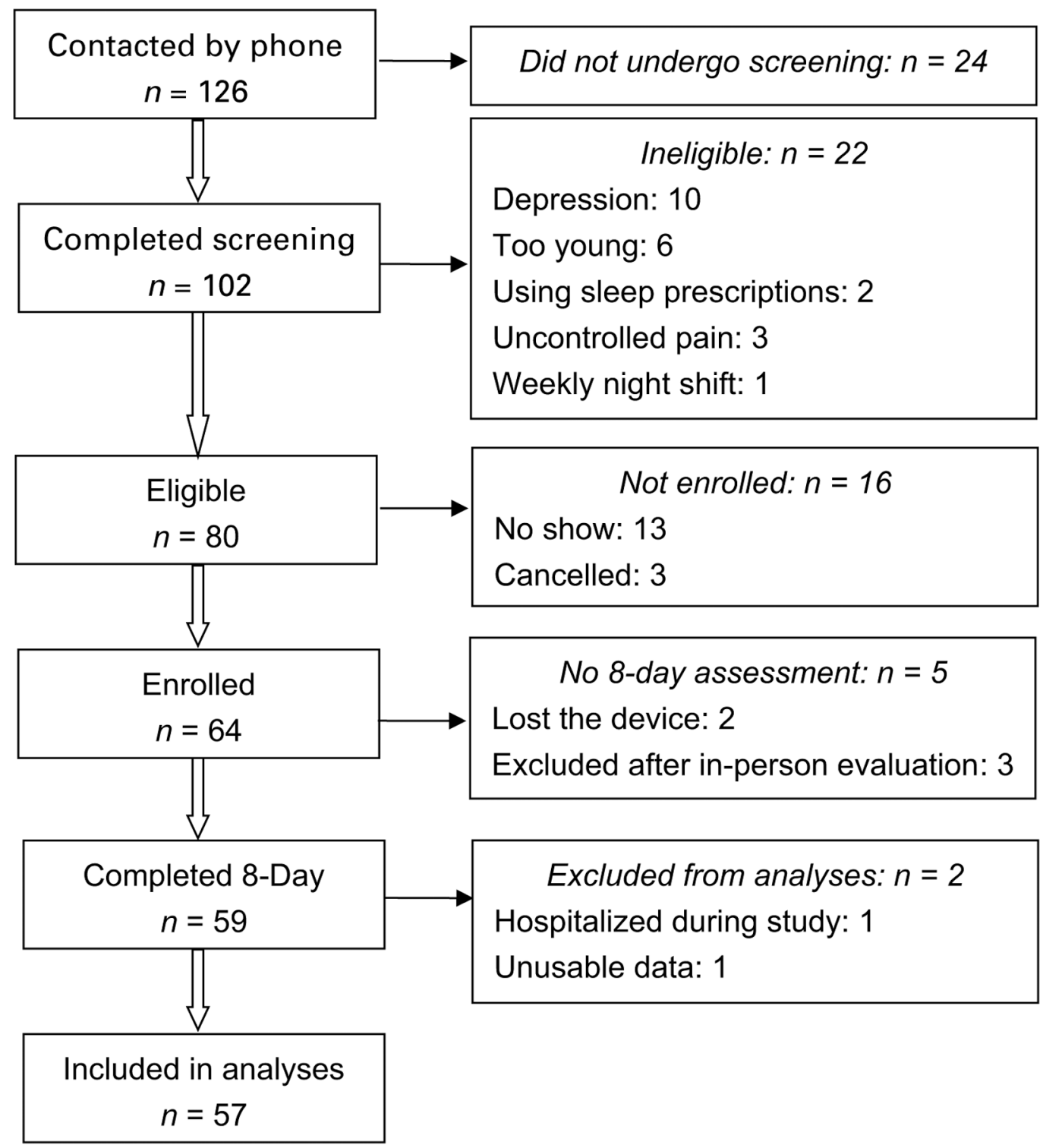

Fig. 1.

Participant recruitment process 


\section{Table 1}

Demographic and health-related characteristics $(n=64)$

\begin{tabular}{|c|c|c|}
\hline Variables & $n(\%) / \bar{x} \pm \mathrm{SD}$ & Range \\
\hline Age & $60.4 \pm 6.8$ & $50-78$ \\
\hline Gender (female) & $33(51.6)$ & \\
\hline \multicolumn{3}{|l|}{ Race/ethnicity } \\
\hline White & $17(26.5)$ & \\
\hline African American & $34(53.1)$ & \\
\hline Hispanic & $12(18.8)$ & \\
\hline Asian & $1(1.6)$ & \\
\hline Education (years) & $14.4 \pm 2.8$ & $4-20$ \\
\hline \multicolumn{3}{|l|}{ Work status } \\
\hline Not working & $37(57.8)$ & \\
\hline Part time & $13(20.3)$ & \\
\hline Full time & $14(21.9)$ & \\
\hline BMI $\left(\mathrm{kg} / \mathrm{m}^{2}\right)$ & $33.8 \pm 8.7$ & $19.2-56.3$ \\
\hline Diabetes duration (years) & $11.2 \pm 8.8$ & $1-40$ \\
\hline HbA1c $(\%)$ & $7.9 \pm 2.0$ & $4.6-13.0$ \\
\hline HbAlc (mmol/mol) & $63 \pm 22$ & $27-119$ \\
\hline Poor glycemic control & $39(61.9)$ & \\
\hline Hypertension (yes) & $38(59.4)$ & \\
\hline \multicolumn{3}{|l|}{ Diabetes treatment regimen } \\
\hline Insulin only & $8(12.5)$ & \\
\hline Oral medication only & $37(57.8)$ & \\
\hline Insulin and oral medication & $14(21.9)$ & \\
\hline Exercise/diet control & $5(7.8)$ & \\
\hline
\end{tabular}




\section{Table 2}

Description of main study variables

\begin{tabular}{lll}
\hline Variables & $\boldsymbol{n}(\boldsymbol{\%}) / \overline{\boldsymbol{x}} \pm \mathrm{SD}$ & Range \\
\hline Self-care & $6.9 \pm 1.5$ & $2.5-10$ \\
Sleep quality (PSQI global score) & $7.0 \pm 3.7$ & $2-20$ \\
$\quad$ Sleep disturbance (PSQI global $>5)$ & $35(54.7)$ & \\
Objective sleep ${ }^{a}$ & & \\
$\quad$ Total sleep time (min) & $394.6 \pm 70.4$ & $250.5-572.4$ \\
$\quad$ Sleep efficiency (\%) & $80.9 \pm 8.1$ & $58.5-94.4$ \\
Sleep onset latency (min) & $7.3 \pm 3.0$ & $4-20.2$ \\
$\quad$ Wake after sleep onset (min) & $87.6 \pm 43.5$ & $21.6-239$ \\
$\quad$ Number of awakenings & $18.7 \pm 7.2$ & $6.9-34.4$ \\
Self-efficacy & $4.1 \pm 0.8$ & $2.3-5$ \\
Diabetes distress & $2.0 \pm 1.0$ & $1-4.7$ \\
Fatigue & $1.6 \pm 1.2$ & $0-4.3$ \\
Daytime sleepiness & $8.7 \pm 4.5$ & $0-24$ \\
\hline
\end{tabular}

${ }^{a} N=57$ 
Table 3

Bivariate correlations between self-care and other variables $(r)$

\begin{tabular}{llll}
\hline & Self-care & & Self-care \\
\hline Age & $0.20^{\dagger}$ & Sleep onset latency & -0.08 \\
Gender & -0.08 & Wake after sleep onset & -0.18 \\
Race & -0.04 & Number of awakenings & $-0.36^{* *}$ \\
Diabetes duration & $0.22^{\dagger}$ & Self-efficacy & $0.33^{* *}$ \\
A1C & $-0.27^{*}$ & Diabetes distress & $-0.53^{* * *}$ \\
Sleep quality & $-0.36^{* *}$ & Fatigue & $-0.39^{* *}$ \\
Total sleep time & -0.02 & Daytime sleepiness & $-0.35^{* *}$ \\
Sleep efficiency & 0.15 & & \\
\hline
\end{tabular}

* $p<0.05$

*** $p<0.01$

*** $p<0.001$

${ }^{\dagger} p<0.20$ 


\section{을
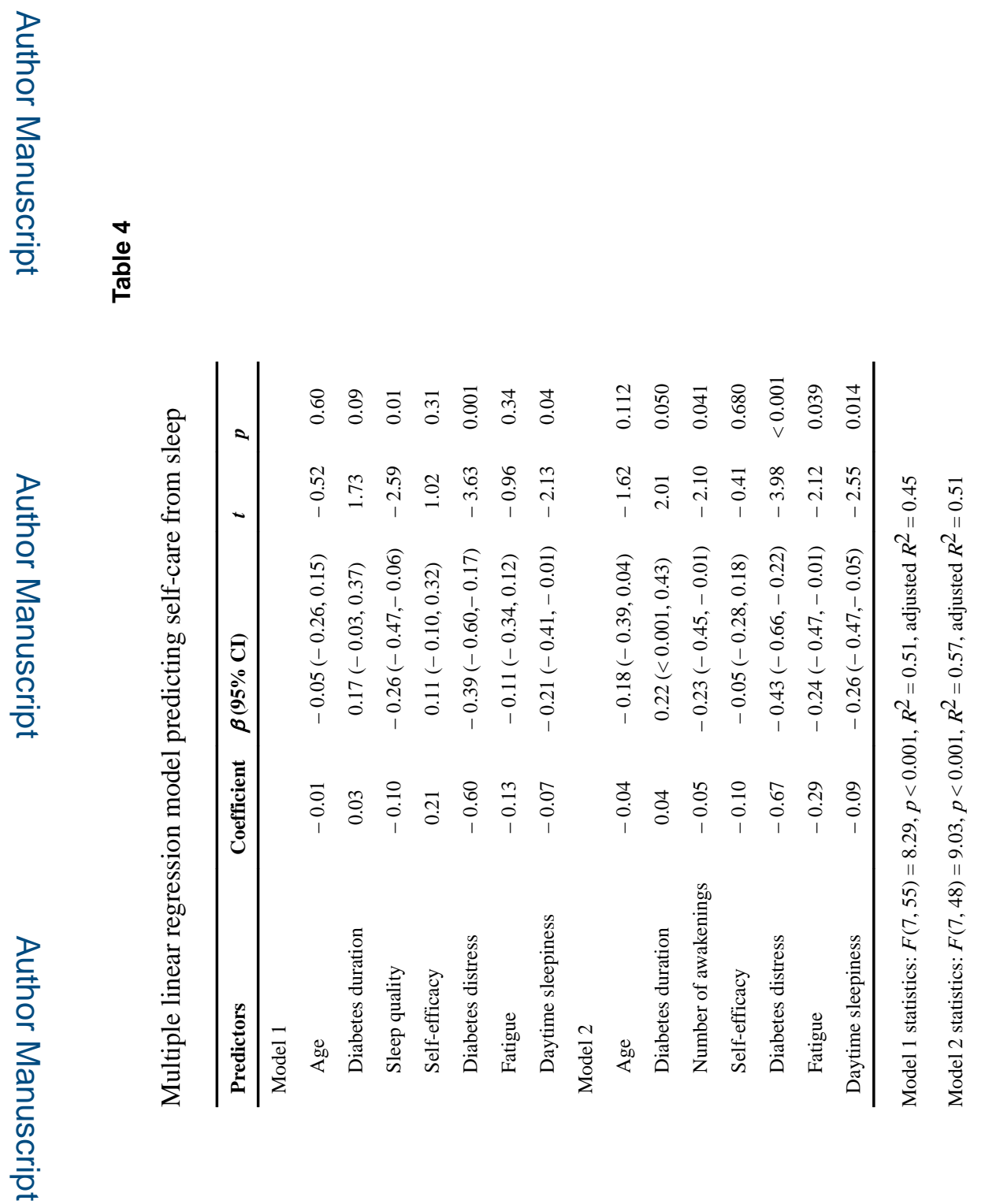